\title{
SOME REMARKS ON THE A POSTERIORI ERROR ANALYSIS OF THE MIXED LAPLACE EIGENVALUE PROBLEM
}

\author{
FLEURIANNE BERTRAND ${ }^{1}$, DANIELE BOFFI ${ }^{2}$, JOSCHA GEDICKE ${ }^{3}$ AND ARBAZ \\ KHAN $^{4}$ \\ ${ }^{1}$ University of Twente
}

Faculty of Electrical Engineering, Mathematics and Computer Science, Zilverling, P.O. Box 217, 7500

AE Enschede, The Netherlands

f.bertrand@utwente.nl

${ }^{2}$ King Abdullah University of Science and Technology (KAUST) and University of Pavia Thuwal 23955-6900, Kingdom of Saudi Arabia and Via Ferrata 1, I-27100 Pavia, Italy daniele.boffi@kaust.edu.sa

${ }^{3}$ University of Bonn

Institute for Numerical Simulation, Friedrich-Hirzebruch-Allee 7, 53115 Bonn, Germany gedicke@ins.uni-bonn.de

\author{
${ }^{4}$ Indian Institute of Technology Roorkee (IITR) \\ Department of Mathematics, Roorkee, India-247667 \\ arbaz@ma.iitr.ac.in
}

Key words: eigenvalue problem, adaptivity, a posteriori error estimator, mixed finite element method, postprocessing

Abstract. In this note we consider the a posteriori error analysis of mixed finite element approximations to the Laplace eigenvalue problem based on local postprocessing. The estimator makes use of an improved $L^{2}$ approximation for the Raviart-Thomas (RT) and Brezzi-Douglas-Marini (BDM) finite element methods. For the BDM method we also obtain improved eigenvalue convergence for postprocessed eigenvalues. We verify the theoretical results in several numerical examples.

\section{INTRODUCTION}

The aim of this note is to discuss some remarks about the approximation of the eigensolutions of the mixed Laplace problem: find $\lambda \in \mathbb{R}$ and $u \in L^{2}(\Omega)$ with $u \neq 0$ such that for some $\sigma \in H(\operatorname{div} ; \Omega)$ it holds

$$
\left\{\begin{aligned}
(\sigma, \tau)+(\operatorname{div} \tau, u) & =0 & & \forall \tau \in H(\operatorname{div} ; \Omega) \\
(\operatorname{div} \sigma, v) & =-\lambda(u, v) & & \forall v \in L^{2}(\Omega) .
\end{aligned}\right.
$$

As it is known, this corresponds to the Dirichlet eigenvalue problem for the Laplace, which in strong 
form reads as follows

$$
\left\{\begin{array}{rlrl}
-\Delta u & =\lambda u & & \text { in } \Omega \\
u=0 & & \text { on } \partial \Omega .
\end{array}\right.
$$

We assume that the reader is familiar with the characterization of the solution to compact symmetric eigenvalue problems and to the classical Babuška-Osborn theory for their finite element approximation. We refer to [5] for a summary, with particular reference to mixed problems.

We are interested in particular in a posteriori error estimates. Several error estimators for the mixed approximation are known for the corresponding source problem, c.f. [1, 9, 7, 8, 11, 13], and in some cases it has been observed how to generalize them to the eigenvalue problem. Residual a posteriori error estimators for the mixed approximation of the Laplace eigenvalue problem have been studied in $[6,10]$, and error estimators based on a local postprocessing are given in [3].

In [3] an error estimator was proposed based on the following two steps: first a local postprocessing procedure was performed in the spirit of [14]; then, the postprocessed solution was averaged in order to obtain a continuous reconstruction of the solution. It was proved that this procedure is effective in the case when Raviart-Thomas (RT) elements are used for the approximation of the gradients. The result relies on the estimation of a typical nonlinear term that arises when arguments valid for the source problem are extended to the eigenvalue problem. In [3] it was shown that such nonlinear contribution is a higher order term with respect to the a priori rate of convergence of the quantities of interest.

In [2] it was shown that the same result doesn't apply to the case when Brezzi-Douglas-Marini (BDM) elements are used for the approximation of ye gradients. Actually, the nonlinear term has been shoyn
to converge at the same order as the other quantities of interest. This implies that the analysis hat
was performed in [3] could not be extended to BDM spaces. This remark was the starting point of this
research that addresses the a posteriori error analysis for the problem under consideration when the BDM
scheme is used.

The eigenvalue problem associated with the Stokes problem was considered in [12] by using a stress-

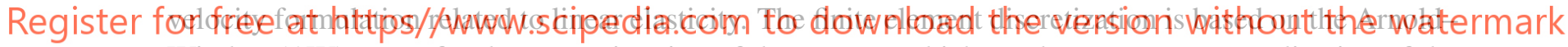
Winther (AW) space for the approximation of the stresses which can be seen as a generalization of the BDM space. For this reason, the results of [12] prove very useful in this context. More precisely, the results translate almost verbatim to the approximation of the mixed Laplacian based on BDM finite elements. It is then quite natural to compare the obtained results with the discussion in [3] and [2]. The a posteriori estimator of [12] is based on the first step only: a local postprocessing procedure in the spirit of [14]. Moreover, it provides in a natural way a postprocessed solution for the eigenvalues which converges faster than the original one.

In this paper we recall the results of [3] for the RT scheme and we state the results of [12] applied to the BDM scheme. 


\section{SETTING OF THE PROBLEM}

The approximation of (1) consists in choosing finite dimensional subspaces $U_{h} \subset L^{2}(\Omega)$ and $\Sigma_{h} \subset$ $H(\operatorname{div} ; \Omega)$ and to seek $\lambda_{h} \in \mathbb{R}$ and $u_{h} \in U_{h}$, with $u_{h} \neq 0$ such that for some $\sigma_{h} \in \Sigma_{h}$ it holds

$$
\left\{\begin{aligned}
\left(\sigma_{h}, \tau\right)+\left(\operatorname{div} \tau, u_{h}\right) & =0 & & \forall \tau \in \Sigma_{h} \\
\left(\operatorname{div} \sigma_{h}, v\right) & =-\lambda_{h}\left(u_{h}, v\right) & & \forall v \in U_{h} .
\end{aligned}\right.
$$

We are going to consider two possible schemes: the RT scheme and the BDM scheme.

We consider a polygonal or polyhedral Lipschitz domain $\Omega$ in $\mathbb{R}^{d}(d=2,3)$ and a conforming triangulation $\mathcal{T}_{h}$ of shape regular triangles or tetrahedra. For $k \geq 0$, in both cases the solution space will be

$$
U_{h}=\left\{v \in U:\left.v\right|_{T} \in P_{k}(T) \forall T \in \mathcal{T}_{h}\right\},
$$

where $P_{k}(T)$ denotes the space of polynomials of degree not exceeding $k$.

In the case of the RT scheme we have

$$
\Sigma_{h}=R T_{k}=\left\{\tau \in H(\operatorname{div} ; \Omega):\left.\tau\right|_{T} \in\left(P_{k}(T)\right)^{d} \oplus \mathbf{x} \tilde{P}_{k}(T) \forall T \in \mathcal{T}_{h}\right\},
$$

where $\tilde{P}_{k}(T)$ is the space of homogeneous polynomials of degree $k$.

For the BDM scheme we have

$$
\Sigma_{h}=B D M_{k+1}=\left\{\tau \in \Sigma:\left.\tau\right|_{T} \in P_{k+1}(T) \forall T \in \mathcal{T}_{h}\right\} .
$$
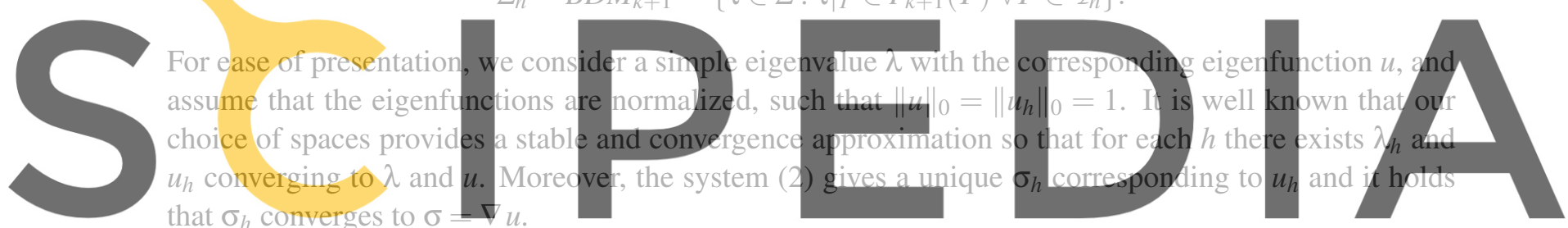

Since $\Omega$ is a polytope, standard regularity results guarantee that the eigenfunction $u$ belongs to $H^{1+s}(\Omega)$,

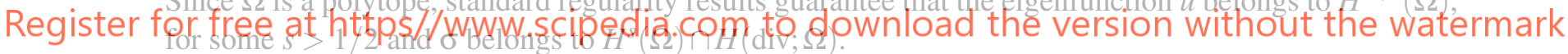

Then, in the case of the RT scheme, the following a priori estimates are satisfied $(k \geq 0)$

$$
\begin{aligned}
\left|\lambda-\lambda_{h}\right| & \leq C h^{2 r}|u|_{1+r} \\
\left\|u-u_{h}\right\|_{0} & \leq C h^{r}|u|_{1+r} \\
\left\|\sigma-\sigma_{h}\right\|_{0} & \leq C h^{r}|u|_{1+r},
\end{aligned}
$$

with $r=\min (s, k+1)$.

When the BDM scheme is used, the effect of a larger space $\Sigma_{h}$ is that the previous estimates become unbalanced. The convergence for $\sigma-\sigma_{h}$ is higher than the other terms and the eigenvalue convergence does not take any advantage by the fact that we are using a richer approximation:

$$
\begin{aligned}
\left|\lambda-\lambda_{h}\right| & \leq C h^{2 r}|u|_{1+r} \\
\left\|u-u_{h}\right\|_{0} & \leq C h^{r}|u|_{1+r} \\
\left\|\sigma-\sigma_{h}\right\|_{0} & \leq C h^{r^{\prime}}|u|_{1+r^{\prime}},
\end{aligned}
$$

where $r=\min (s, k+1)$ as before and $r^{\prime}=\min (s, k+2)$. If the solution is smooth enough, then $r^{\prime}=r+1$. 


\section{THE POSTPROCESSING TECHNIQUE}

For $k \geq 0$ and $\ell \geq 1$ we consider the space

$$
U_{h}^{*}=\left\{v \in L^{2}(\Omega):\left.v\right|_{T} \in P_{k+\ell}(T) \forall T \in \mathcal{T}_{h}\right\} .
$$

The local postprocessing of the eigenfunction $u_{h} \in U_{h}$ can be performed along the lines of $[13,14]$ as follows: find $u_{h}^{*} \in U_{h}^{*}$ such that

$$
\left\{\begin{aligned}
P_{h} u_{h}^{*} & =u_{h} \\
\left(\nabla u_{h}^{*}, \nabla v\right)_{T} & =\left.\left(\sigma_{h}, \nabla v\right) \quad \forall v \in\left(I-P_{h}\right) U_{h}^{*}\right|_{T} \forall T \in \mathcal{T}_{h},
\end{aligned}\right.
$$

where $P_{h}$ denotes the $L^{2}(\Omega)$-projection onto $U_{h}, I$ is the identity operator, and $\sigma_{h}$ is the component of the solution associated with $u_{h}$.

A variational formulation of the construction of $u_{h}^{*}$ is obtained, for instance, by solving the following local problem for all $T$ in $\mathcal{T}_{h}$ : find $\left.u_{h}^{*} \in U_{h}^{*}\right|_{T}$ and $\left.z_{h} \in U_{h}\right|_{T}$ such that

$$
\left\{\begin{aligned}
\left(\nabla u_{h}^{*}, \nabla v\right)+\left(z_{h}, v\right) & =\left(\sigma_{h}, \nabla v\right) & & \left.\forall v \in U_{h}^{*}\right|_{T} \\
\left(u_{h}^{*}, w\right) & =\left(u_{h}, w\right) & & \left.\forall w \in U_{h}\right|_{T} .
\end{aligned}\right.
$$

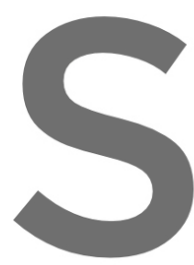

The choice of $\ell$ depends

and in the case of BDM

should be mimicking of

higher rate of convergence

3.1 Postprocessing of the eigenvalue for the BDM scheme
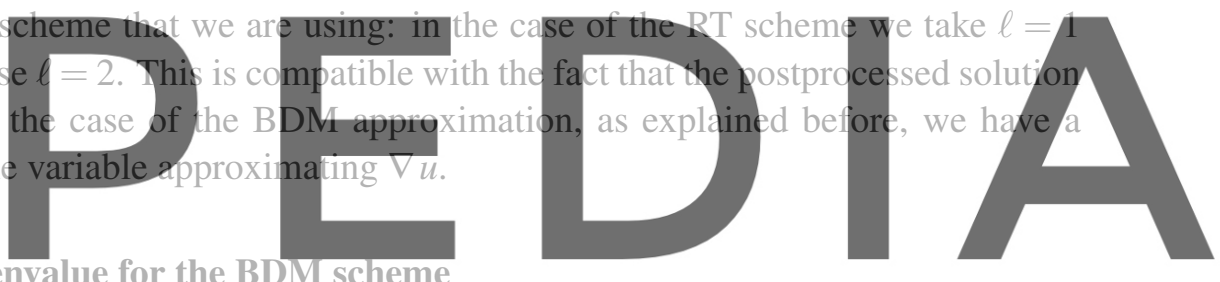

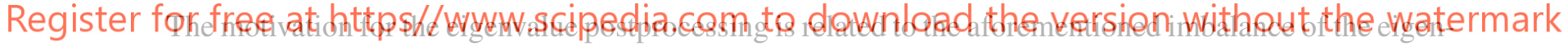

function errors. The identity [10, Lemma 3.2] for the eigenvalue and eigenfunction errors

$$
\lambda-\lambda_{h}=\left\|\sigma-\sigma_{h}\right\|_{0}^{2}-\lambda_{h}\left\|u-u_{h}\right\|_{0}^{2}
$$

shows that the convergence of the eigenvalues is limited by the $L^{2}$ convergence of the eigenfunctions. Hence, we may improve the eigenvalue convergence by a postprocessing of both the eigenfunction $u_{h}$ and the eigenvalue $\lambda_{h}$.

It can be easily seen that the eigenvalues $\lambda_{h}$ of (2) are related to $u_{h}$ and $\sigma_{h}$ by the following relation which resembles the Rayleigh quotient

$$
\lambda_{h}=-\frac{\left(\operatorname{div} \sigma_{h}, u_{h}\right)}{\left\|u_{h}\right\|_{0}^{2}} .
$$

Following [12, Def. 4.2] it is then natural to define a postprocessed eigenvalue by replacing $u_{h}$ with the postprocessed solution $u_{h}^{*}$

$$
\lambda_{h}^{*}=-\frac{\left(\operatorname{div} \sigma_{h}, u_{h}^{*}\right)}{\left\|u_{h}^{*}\right\|_{0}^{2}} .
$$


It turns out that the postprocessed eigenvalue satisfies the following bound

$$
\left|\lambda-\lambda_{h}^{*}\right| \leq C\left(\left\|\sigma-\sigma_{h}\right\|_{0}^{2}+\left\|u-u_{h}^{*}\right\|_{0}^{2}+\left\|\operatorname{div}\left(\sigma-\sigma_{h}\right)\right\|_{0}\left\|u-u_{h}^{*}\right\|_{0}+\left|\lambda-\lambda_{h}^{*}\right|^{2}\right),
$$

that can be obtained in the same way as in [12, Thm. 4.3]. This implies the following superconvergence result if the solution is smooth enough.

Theorem 1. Let $u$ be in $H^{k+2}(\Omega)$ and $\sigma$ in $H^{k+2}(\Omega)$, then the following estimate is valid

$$
\left|\lambda-\lambda_{h}^{*}\right| \leq C h^{2(k+2)}\left(\|u\|_{k+2}+\|\sigma\|_{k+2}\right) .
$$

\subsection{Error estimator for the RT scheme}

The error estimator in the case of the RT is obtained in [3] by performing a further postprocessing of the function $u_{h}^{*}$. It consists in a standard averaging technique that returns a function $u_{h}^{* *}$ in $H_{0}^{1}(\Omega)$. More precisely, using Oswald interpolation, it is possible to define $u_{h}^{* *}$ in the space

$$
U_{h}^{* *}=\left\{v \in H_{0}^{1}(\Omega):\left.v\right|_{T} \in P_{k+1}(T) \forall T \in \mathcal{T}_{h}\right\}
$$

\section{Satisfying}

\section{$\left\|\nabla u-\nabla u_{h}^{* *}\right\|_{0} \leq C h^{r}\|u\|_{1+r}$}

\section{with $r=\min (s, k+1)$.}

A natural error estimator can then be defined as
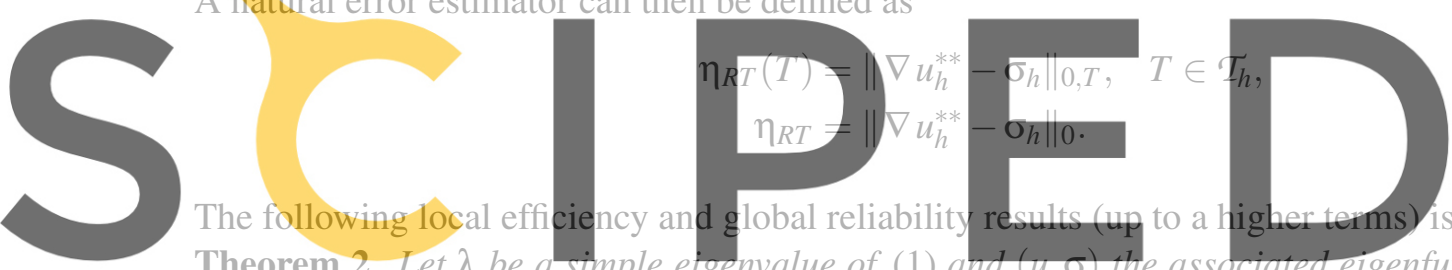

Theorem 2. Let $\lambda$ be a simple etgenvalue of (1) a rat

corresponding discrete eigenvalue of $(1)$ and $\left(u_{h}, \sigma_{h}\right)$ converging to $(u, \sigma)$ when the $R T$ scheme is used.

Register for free at https//Www. Scipedia.com to download the version without the watermark

$$
\eta_{R T}(T) \leq\left\|\nabla i-\nabla i_{h}^{* *}\right\|_{0, T}+\left\|\sigma-\sigma_{h}\right\|_{0, T} .
$$

Moreover, the following global reliability is satisfied

$$
\left\|\nabla u-\nabla u_{h}^{* *}\right\|_{0}^{2}+\left\|\sigma-\sigma_{h}\right\|_{0}^{2} \leq \eta_{R T}^{2}+h o t(h)
$$

with

$$
\operatorname{hot}(h)=2\left\|u-u_{h}^{* *}\right\|_{0}\left\|\lambda u-\lambda_{h} u_{h}\right\|_{0} .
$$

The previous theorem has the merit of providing an a posteriori analysis with explicit constants equal to one in the efficiency and reliability bounds. It could be extended to the BDM scheme as well, but in this case the term hot $(t)$ is not of higher order. Numerical experiments in [2] show that indeed it is of the same order as the estimator.

By using the identity (3) and the previous theorem, we easily obtain the following reliability result for the error in the eigenvalue when a slightly modified error estimator is used. 
Corollary 1 (reliability for the error in the eigenvalue). Under the same hypotheses of the previous theorem, for $h$ small enough it holds

$$
\left|\lambda-\lambda_{h}\right| \leq \tilde{\eta}_{R T}^{2}+h o t(h)
$$

with

$$
\tilde{\eta}_{R T}^{2}:=\eta_{R T}^{2}+2 \lambda_{h}\left\|u_{h}^{* *}-u_{h}\right\|_{0}^{2}, \quad h o t(h):=2 \lambda_{h}\left\|u-u_{h}^{* *}\right\|_{0}^{2}+2\left\|u-u_{h}^{* *}\right\|_{0}\left\|\lambda u-\lambda_{h} u_{h}\right\|_{0} .
$$

In the next subsection we show how the estimator introduced in [12] for the Stokes eigenproblem can be used for the BDM scheme applied to (2).

\subsection{Error estimator for the BDM scheme}

For the BDM scheme we define the following error indicator which is based on the postprocessed eigenvalue $\lambda_{h}^{*}$ and eigenfunction $u_{h}^{*}$ without the need of any additional regularizations

$$
\eta_{B D M}^{2}=\sum_{T \in \mathcal{T}_{h}}\left\|\nabla u_{h}^{*}-\sigma_{h}\right\|_{0, T}^{2}+\sum_{T \in \mathcal{T}_{h}} h_{T}^{2}\left\|\lambda_{h}^{*} u_{h}^{*}+\operatorname{div} \sigma_{h}\right\|_{0, T}^{2}+\sum_{E \in \mathcal{E}_{h}} h_{E}^{-1}\left\|\llbracket u_{h}^{*} \rrbracket\right\|_{0, E}^{2} .
$$

We now state some reliability and efficiency theorems that are obtained analogously to what has been done in [12]. We don't repeat the proofs, but we give reference to the corresponding results in [12] from which the proofs can be obtained with natural modifications.

Theorem 3 (reliability simple eigenvalue of ( eigenvalue of (1) and estimate holds true
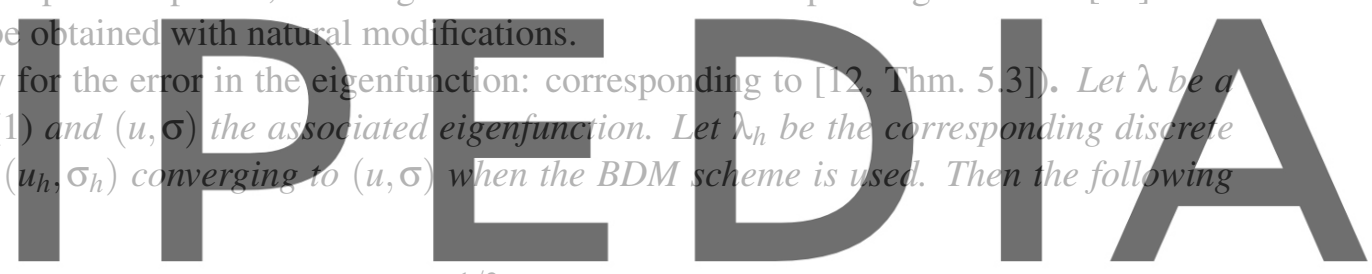

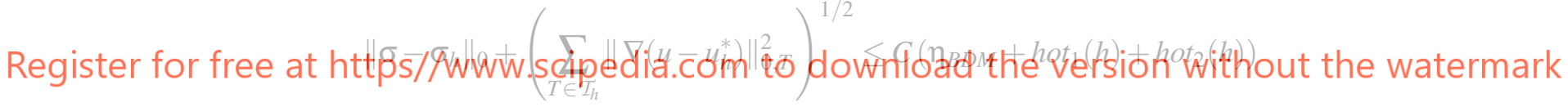
with

$$
\operatorname{hot}_{1}(h)=\left(\sum_{T \in \mathcal{T}_{h}} h_{T}^{2}\left\|\lambda u-\lambda_{h}^{*} u_{h}^{*}\right\|_{0, T}^{2}\right)^{1 / 2}, \quad \operatorname{hot}_{2}(h)=\lambda_{h}\left\|u-u_{h}^{*}\right\|_{0}+\left|\lambda-\lambda_{h}\right| .
$$

From [14, Thm 2.2] we have for the postprocessing of the BDM scheme applied to the associated source problem with source term $f=\lambda u$ satisfies the following bound

$$
\left\|u-u_{h}^{*}\right\|_{0} \leq \begin{cases}C h^{1+r^{\prime}}\left(|\sigma|_{r^{\prime}}+|u|_{1+r^{\prime}}\right) & \text { for } k \geq 1 \\ C h^{2}\left(|\sigma|_{2}+|u|_{2}\right) & \text { for } k=0 .\end{cases}
$$

One can show that the same estimates hold true also for the eigenvalue problem. Hence, $h_{0} t_{1}(h)$ is of higher order $O\left(h^{1+r^{\prime}}\right)$ for all $k \geq 0$. For the second term $h_{0} t_{2}(h)$ one has to distinguish two cases: for $k \geq 1, h_{2}(h)$ is of higher order $O\left(h^{1+r^{\prime}}\right)$, while for $k=0$ it is of the same order $O\left(h^{2}\right)$. However, $\eta_{B D M}$ can still be used as a posteriori error estimator for $k=0$ in practice, as demonstrated in the numerical examples of Section 4. 

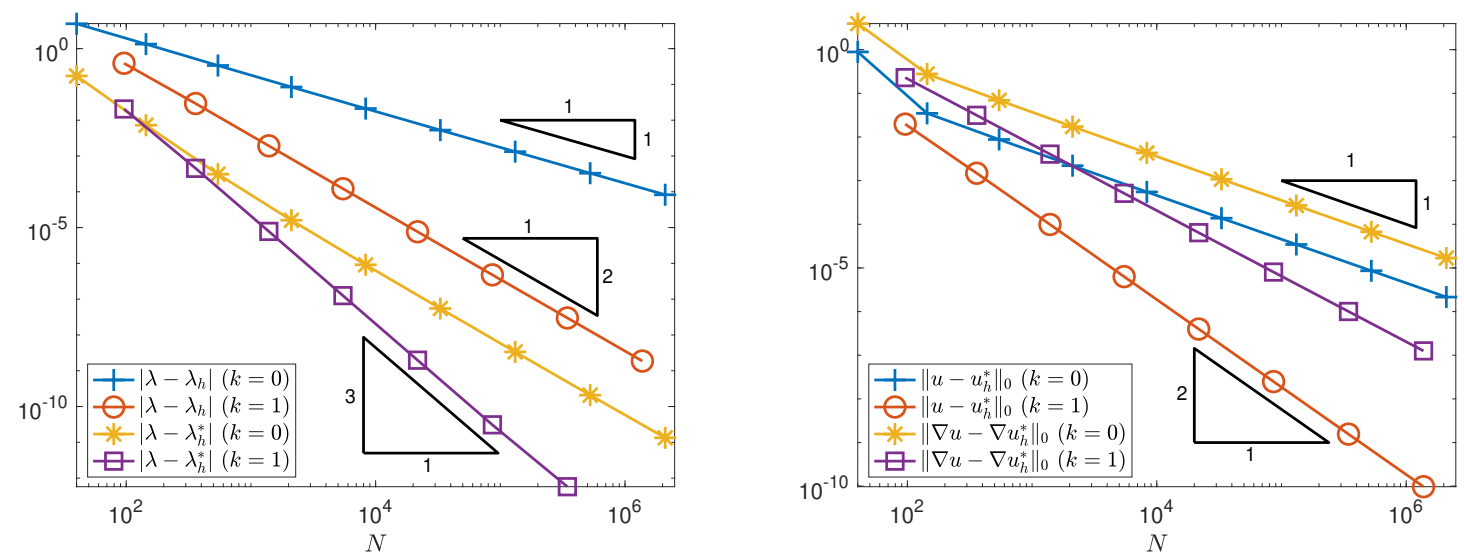

Figure 1: Convergence of the BDM postprocessed eigenvalues (left) and eigenfunctions (right) on the unit square

Theorem 4 (reliability for the error in the eigenvalue: corresponding to [12, Thm. 5.4]). Under the same hypotheses of the previous theorem, for h small enough it holds

$$
\left|\lambda-\lambda_{h}^{*}\right| \leq C\left(\eta_{B D M}^{2}+\left(h_{1}(h)+\operatorname{hot}_{2}(h)\right)^{2}\right) .
$$

Theorem 5 (efficiency: corresponding to [12, Thm. 5.5]). Under the same assumptions as in the previous theorem, the following bou
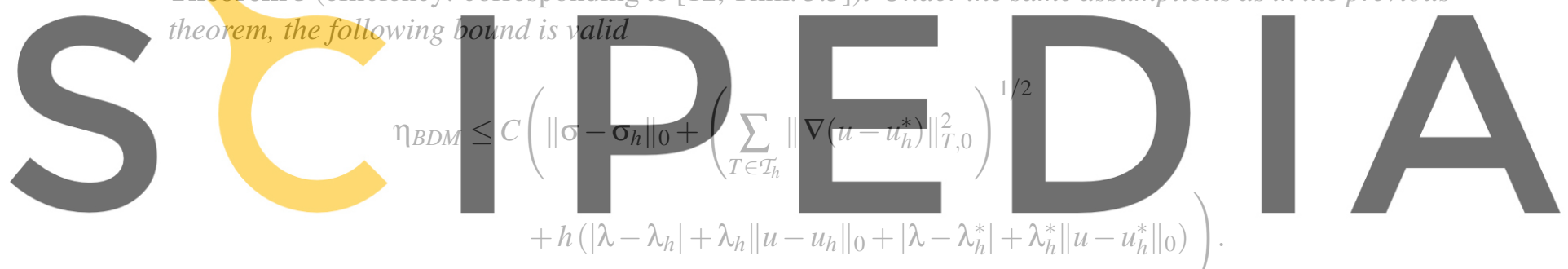

Register for free at https//www.scipedia.com to download the version without the watermark

Note that while reliability holds for any $u_{h}^{*} \in U_{h}^{*}, k \geq 1$, efficiency holds only for convex domains with sufficiently smooth solutions. However, as we will demonstrate in the next section, efficiency also holds numerically in the case of singular eigenfunctions for graded meshes generated by an adaptive algorithm.

\section{NUMERICAL EXPERIMENTS}

This section is devoted to several numerical experiments using uniform and adaptive $h$-refinements of triangular meshes for two-dimensional domains. The solution of problem (2) is computed with RT and BDM elements of order $k=0$ and $k=1$.

\subsection{Unit square}

As first example we consider the unit square $\Omega=(0,1)^{2}$. The first eigenvalue $\lambda=2 \pi^{2}$ is known to be simple and the corresponding eigenfunction ( $L^{2}$-normalized to 1$)$ reads $u(x)=2 \sin \left(\pi x_{1}\right) \sin \left(\pi x_{2}\right)$. In Figure 1 we verify the higher order convergence of the postprocessed eigenvalues $\lambda_{h}^{*}$ and eigenfunctions 

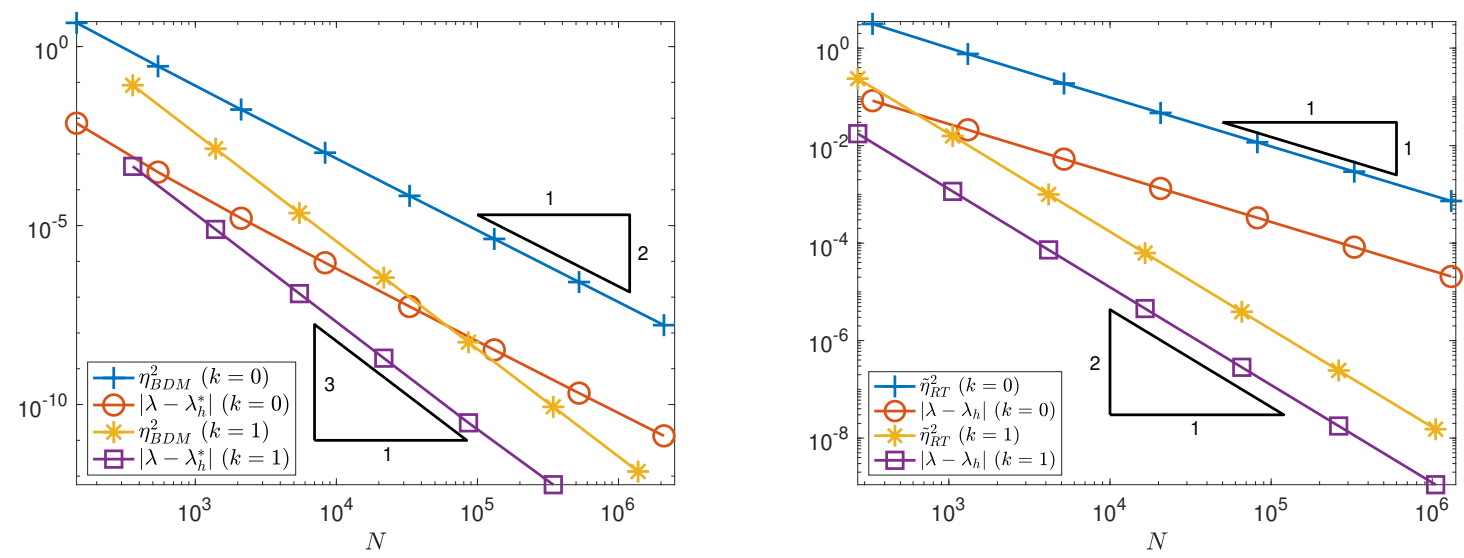

Figure 2: Convergence of eigenvalues and estimators for BDM (left) and RT (right) on the unit square
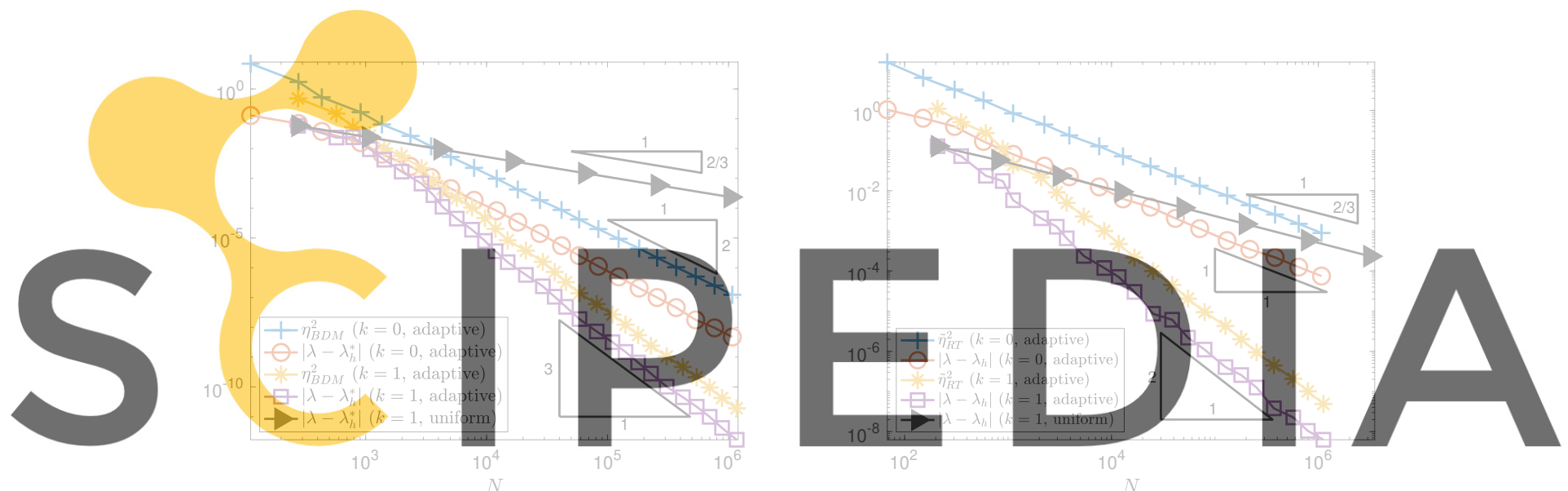

Register for free at https//www.scipedia.com to download the version without the watermark Figure 3: Convergence of eigenvalues and estimators for BDM (left) and RT (right) on the L-shaped domain

$u_{h}^{*}$ for the BDM scheme. Note that for uniform refinement $O(h)=O\left(N^{-1 / 2}\right)$, where $N:=\operatorname{dim}\left(\Sigma_{h}\right)+$ $\operatorname{dim}\left(U_{h}\right)$ denotes the number of degrees of freedom. Hence, we observe $O\left(h^{2(k+2)}\right)$ convergence of the postprocessed eigenvalues $\lambda_{h}^{*}$ as predicted by the theory. For the postprocessed eigenfunctions $u_{h}^{*}$ we observe $O\left(h^{k+2}\right)$ convergence in the $H^{1}$-norm, and for $k=1, O\left(h^{k+3}\right)$ convergence in the $L^{2}$-norm. Note that in the case $k=0$, the convergence of $u_{h}^{*}$ in the $L^{2}$-norm is only of order $O\left(h^{2}\right)$ as predicted by the theory.

In Figure 2 we demonstrate the reliability and efficiency of $\eta_{B D M}^{2}$ and $\tilde{\eta}_{R T}^{2}$ for $k=0,1$ and the eigenvalue error. Due to the improved eigenvalue convergence, the BDM scheme yields faster convergence for the same index $k$ as the RT scheme. The estimator $\eta_{B D M}^{2}$ shows to be reliable and efficient also in the case $k=0$. 


\subsection{L-shaped domain}

The first eigenvalue for the L-shaped domain $\Omega=(-1,1)^{2} \backslash([0,1] \times[-1,0])$ reads $\lambda \approx 9.6397238440219$, where all but the last two digits have been proven to be correct [4]. Since the corresponding eigenfunction is singular, we observe suboptimal convergence rates of $O\left(N^{-2 / 3}\right)$ for uniform meshes in Figure 3. We can improve the order of convergence using an adaptive finite element scheme. We consider the standard algorithm with the steps solve, estimate, mark and refine. We employ a direct solver to solve the saddle point systems, the bulk marking strategy with bulk parameter $\theta=1 / 2$, and the red-green-blue refinement strategy. The estimators $\eta_{B D M}^{2}$ and $\tilde{\eta}_{R T}^{2}$ prove to be reliable and efficient for the eigenvalue error on adaptively refined meshes. Moreover, we observe optimal orders of convergence $O\left(N^{-(k+2)}\right)$ for the BDM adaptive scheme with postprocessed eigenvalues and $O\left(N^{-(k+1)}\right)$ for the RT adaptive scheme.

\section{ACKNOWLEDGEMENTS}

Fleurianne Bertrand gratefully acknowledges support by the German Research Foundation (DFG) in the Priority Programme SPP 1748 Reliable simulation techniques in solid mechanics under grant number BE6511/1-1. Daniele Boffi is member of the INdAM Research group GNCS and his research is partially supported by IMATI/CNR and by PRIN/MIUR. Arbaz Khan has been supported by the faculty initiation grant MTD/FIG/100878 and Serb Matrics grant MTR/2020/000303.

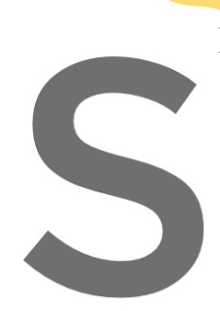

REFERENCES
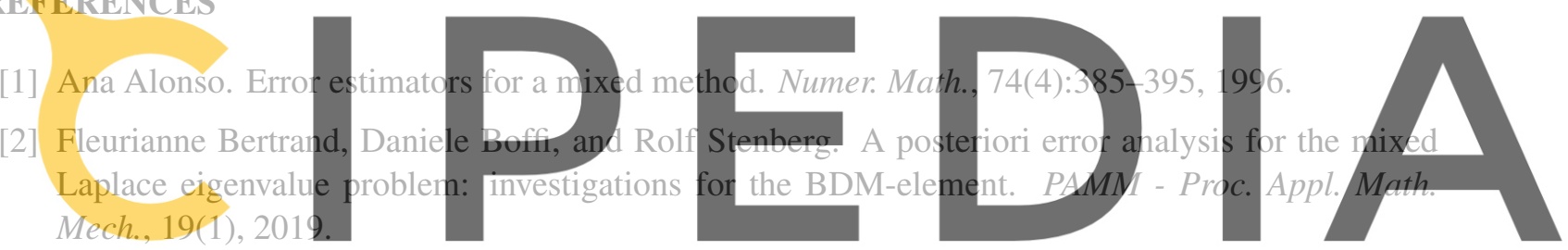

[3] Fleurianne Bertrand, Daniele Boffi, and Rolf Stenberg. Asymptotically exact a posteriori error

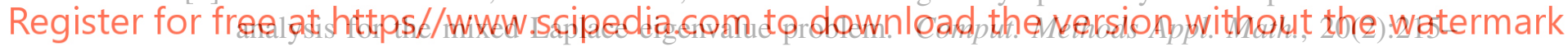
225, 2020.

[4] Timo Betcke and Lloyd N. Trefethen. Reviving the method of particular solutions. SIAM Rev., 47(3):469-491, 2005.

[5] Daniele Boffi. Finite element approximation of eigenvalue problems. Acta Numer., 19:1-120, 2010.

[6] Daniele Boffi, Dietmar Gallistl, Francesca Gardini, and Lucia Gastaldi. Optimal convergence of adaptive FEM for eigenvalue clusters in mixed form. Math. Comp., 86(307):2213-2237, 2017.

[7] Carsten Carstensen. A posteriori error estimate for the mixed finite element method. Math. Comp., 66(218):465-476, 1997.

[8] Carsten Carstensen and Sören Bartels. Each averaging technique yields reliable a posteriori error control in FEM on unstructured grids. I. Low order conforming, nonconforming, and mixed FEM. Math. Comp., 71(239):945-969, 2002.

[9] Carstensen Carstensen. A unifying theory of a posteriori finite element error control. Numer. Math., 100(4):617-637, 2005. 
[10] Ricardo G. Durán, Lucia Gastaldi, and Claudio Padra. A posteriori error estimators for mixed approximations of eigenvalue problems. Math. Models Methods Appl. Sci., 9(8):1165-1178, 1999.

[11] Alexandre Ern and Martin Vohralík. Polynomial-degree-robust a posteriori estimates in a unified setting for conforming, nonconforming, discontinuous Galerkin, and mixed discretizations. SIAM J. Numer. Anal., 53(2):1058-1081, 2015.

[12] Joscha Gedicke and Arbaz Khan. Arnold-Winther mixed finite elements for Stokes eigenvalue problems. SIAM J. Sci. Comput., 40(5):A3449-A3469, 2018.

[13] Carlo Lovadina and Rolf Stenberg. Energy norm a posteriori error estimates for mixed finite element methods. Math. Comp., 75(256):1659-1674, 2006.

[14] Rolf Stenberg. Postprocessing schemes for some mixed finite elements. RAIRO Modél. Math. Anal. Numér., 25(1):151-167, 1991.
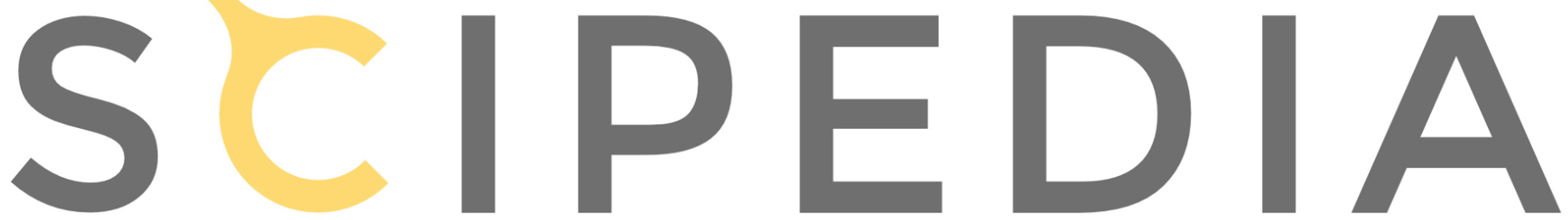

Register for free at https//www.scipedia.com to download the version without the watermark 